\title{
Spatial Frequency Discrimination of Band-limited Periodic Targets: Effects of Stimulus Contrast, Bandwidth and Retinal Eccentricity
}

\author{
MARK W. GREENLEE* \\ Received 20 February 1991; in revised form 9 July 1991
}

\begin{abstract}
Two experiments were conducted to explore the ability of human observers to discriminate the spatial frequency of briefly-presented, Gaussian-truncated sinewave gratings. In the first experiment, the influence of stimulus contrast and stimulus bandwidth on discrimination thresholds was measured after removing any position cues by randomizing the spatial phase of the gratings for each presentation. In a second experiment, the influence of retinal eccentricity on discrimination thresholds was explored for Gaussian-truncated gratings of constant spatial frequency bandwidth (0.5 octave) and suprathreshold contrast value ( $5 \times$ detection threshold). The spatial frequency of the reference gratings varied from 1 to $8 \mathrm{c} / \mathrm{deg}$. The gratings were positioned centered at the fixation point or 1-20 deg eccentric of the point of fixation along the horizontal meridian. Two observers responded in a two-interval forced-choice paradigm, which of two gratings had a higher spatial frequency. $A$ difference frequency was randomly added to or subtracted from the spatial frequency of either the first or second grating. Using a maximum-likelihood algorithm, the spatial-frequency discrimination threshold $\Delta f$ was computed from 40 trials, at which the observer responded with $75 \%$ accuracy. The results indicate that discrimination thresholds increase with (1) decreasing stimulus contrast, (2) increasing stimulus bandwidth, and (3) increasing retinal eccentricity. It is shown that spatial-frequency discrimination thresholds are only independent of contrast for narrow bandwidth stimuli having a contrast $>0.02$. The eccentricity-dependent increase in discrimination thresholds varies with reference spatial frequency: with increasing retinal eccentricity $\Delta f / f$ increases gradually for low spatial frequencies but rapidly for high spatial frequencies.
\end{abstract}

Spatial frequency discrimination Contrast Bandwidth Retinal eccentricity

\section{INTRODUCTION}

The human observer is capable of making fine discriminations between sinewave gratings which differ by as little as $3 \%$ in their spatial frequency (Campbell, Nachmias \& Jukes, 1970; Hirsch \& Hylton, 1982; Meyer \& Kim, 1986). Such low discrimination thresholds have resulted from experiments in which two large-field gratings were presented either side-by-side or sequentially. Near detection threshold, however, discrimination thresholds increase (Watson \& Robson, 1981; Thomas, 1983). More recently it has been shown that spatialfrequency, temporal-frequency and orientation discrimination thresholds become independent of stimulus contrast whenever contrast is a few times greater than

\footnotetext{
*Neurologische Universitätsklinik, Abteilung für Neurophysiologie, Universität Freiburg, Hansastr. 9, 7800 Freiburg, Fed. Rep. Germany.
}

detection threshold, while contrast discrimination continues to improve with increasing pedestal contrast (Gouled Smith \& Thomas, 1989; Bowne, 1990). To explain the discrepancy between contrast discrimination and spatial-frequency or orientation discrimination Gouled Smith and Thomas (1989) suggest that the underlying transducer function saturates at low contrast levels, and that the noise associated with the stimulus is independent of contrast. Bowne (1990), on the other hand, argues that a compressive contrast transducer function alone is insufficient to account for the independence of performance from stimulus contrast. $\mathrm{He}$ suggests rather that what he refers to as "central noise" must be added at the stage where the outputs of different neural mechanisms are compared.

It is now well documented that visual performance changes with increasing retinal eccentricity (Weymouth, 1958; Virsu \& Rovamo, 1979; Westheimer, 1982; Levi, Klein \& Aitsebaomo, 1985; Rentschler \& Treutwein, 
1985; Bennett \& Banks, 1987; for a recent review see Wilson, Levi, Maffei, Rovamo \& DeValois, 1990). Thomas (1987) has reported that the ratio between the normalized probability that a grating is correctly identified and/or detected (I/D ratio) can depend on retinal eccentricity, and that these ratios vary for different spatial frequencies at different retinal eccentricities.

Two experiments have been conducted to explore the dependence of spatial-frequency discrimination thresholds on stimulus contrast, spatial-frequency bandwidth, and retinal eccentricity. The findings of the first experiment suggest that the contrast independency of spatial-frequency discrimination described by Bowne (1990) is due to a "floor effect" which is related to the use of narrow-band periodic gratings. The findings of the second experiment indicate that the spatial-frequency discrimination threshold increases with increasing retinal eccentricity and that the rate of this change in threshold depends on the reference spatial frequency.

\section{METHOU}

\section{Stimuli}

Sinewave luminance gratings of vertical orientation were produced on a high-resolution display (Joyce Electronics), having a white (P4) phosphor, a frame rate of $100 \mathrm{~Hz}$ and an average mean luminance of $200 \mathrm{~cd} / \mathrm{m}^{2}$. The linearity of the control voltage-luminance characteristic of the display was calibrated using a spot photometer. The contrast of the grating stimuli was modulated in space and time by Gaussian envelopes (cf. Marcelja, 1980). The luminance distribution of the gratings orthogonal to the gratings orientation can be described by the equation:

$$
\begin{aligned}
& L(x)=L_{\mathrm{o}}\left(1+C\left[\operatorname { s i n } \left\{2 \pi f_{0}\left(x-x_{0}\right)\right.\right.\right. \\
& +\varphi \text { random }\}] \exp \left(\frac{-\left(x-x_{0}\right)^{2}}{2 \sigma_{x}^{2}}\right) \exp \left(\frac{-\left(t-t_{\mathrm{o}}\right)^{2}}{2 \sigma_{t}^{2}}\right) .
\end{aligned}
$$

In the temporal domain, the Gaussian envelope had a standard deviation $\sigma_{t}$ of $100 \mathrm{msec}$; the entire duration was thus approx. $600 \mathrm{msec}$. In the space domain the standard deviation $\sigma_{x}$ of the Gaussian was varied between 0.078 and $0.54 \mathrm{deg}$ in separate measurements. Contrast was defined by the Michelson equation:

$$
C=\left(L_{\max }-L_{\min }\right) /\left(L_{\max }+L_{\min }\right) \text {. }
$$

Figure 1 presents schematically examples of the test stimuli used. Four different spatial-frequency bandwidths $(0.25,0.5,1$ and 2 octaves) are shown for Gabor-type stimuli with a center spatial frequency of $4 \mathrm{c} /$ deg.

The contrast of the gratings varied from 0.01 to 0.16 . In a separate set of measurements in which we explored the effect of the reference spatial frequency, the contrast of the grating was adjusted to be 5 times greater than detection threshold for each stimulus condition tested. To eliminate position cues, the spatial phase of the gratings was randomly determined for each stimulus presentation. Although this manipulation rules out the use of position cues to facilitate correct discrimination, the maximum and minimum luminance level of the gratings (and thus the Michelson contrast) varied with spatial phase. This manipulation had the effect that the perceived contrast of the grating could not be used as additional information, since it also varied somewhat with the (random) spatial phase of the grating.

\section{Procedure: Experiment 1}

Observers binocularly viewed the display at a distance of $2.28 \mathrm{~m}$. Throughout this experiment, the gratings were presented centered at the point of fixation. The display, which subtended $7.5 \times 5 \mathrm{deg}$, was surrounded by a semicircular, back-illuminated Plexiglass screen, which had a mean luminance of $100 \mathrm{~cd} / \mathrm{m}^{2}$. The mean luminance of the display was $200 \mathrm{~cd} / \mathrm{m}^{2}$. Viewing distance and head orientation was held constant by having observers place their chin on a chin-forehead rest. The observers responded to a two-interval forced-choice paradigm which of two gratings had the higher spatial frequency. Each experimental run consisted of 5 stimuli which varied in their contrast level but were constant in their reference spatial frequency and average spatialfrequency bandwidth. The program controlling the experiment and recording the responses randomly selected, on a given trial, which of five contrast levels was presented and whether a spatial-frequency increment or decrement was given. The spatial-frequency increment/decrement was added on a random basis to the spatial frequency of either the first or second grating. The observer had to attend to both the first and second grating as there was $50 \%$ uncertainty as to which was the reference $(f)$ and test grating $(f+\Delta f$ or $f-\Delta f)$. The task only demanded that the observer judge the relative spatial frequency of the two gratings. A total of 200 trials (5 stimuli, 40 trials each) constituted a single run. The results shown in Figs 2-5 show the mean values of three such independent runs, which were conducted on different days.

Frequency discrimination thresholds were estimated using a staircase technique that controlled the value of $\Delta f$, which could range from 1 to $20 \%$ in increments of $1 \%, 2$ to $40 \%$ with increments of $2 \%$, or 20 to $60 \%$ with increments of $2 \%$ of the reference spatial frequency. The different ranges of $\Delta f$ were used in conjunction with the different spatial frequency bandwidth conditions. A maximum likelihood algorithm (Best-PEST, Lieberman \& Pentland, 1982) was used to provide an efficient estimate of the threshold, which was defined as the $75 \%$ correct performance level. The maximum likelihood estimation was determined for each of 40 trials, the estimate given after the 40 th trial was defined as the threshold measure. The final standard deviation of the likelihood function calculated within a single run usually varied from $\Delta f / f=0.02$ to $\Delta f / f=0.04$ (i.e. $2-4$ steps of the 20 step staircase). Threshold estimates were rejected if the standard deviation exceeded 4 steps, which only occurred seldomly. 


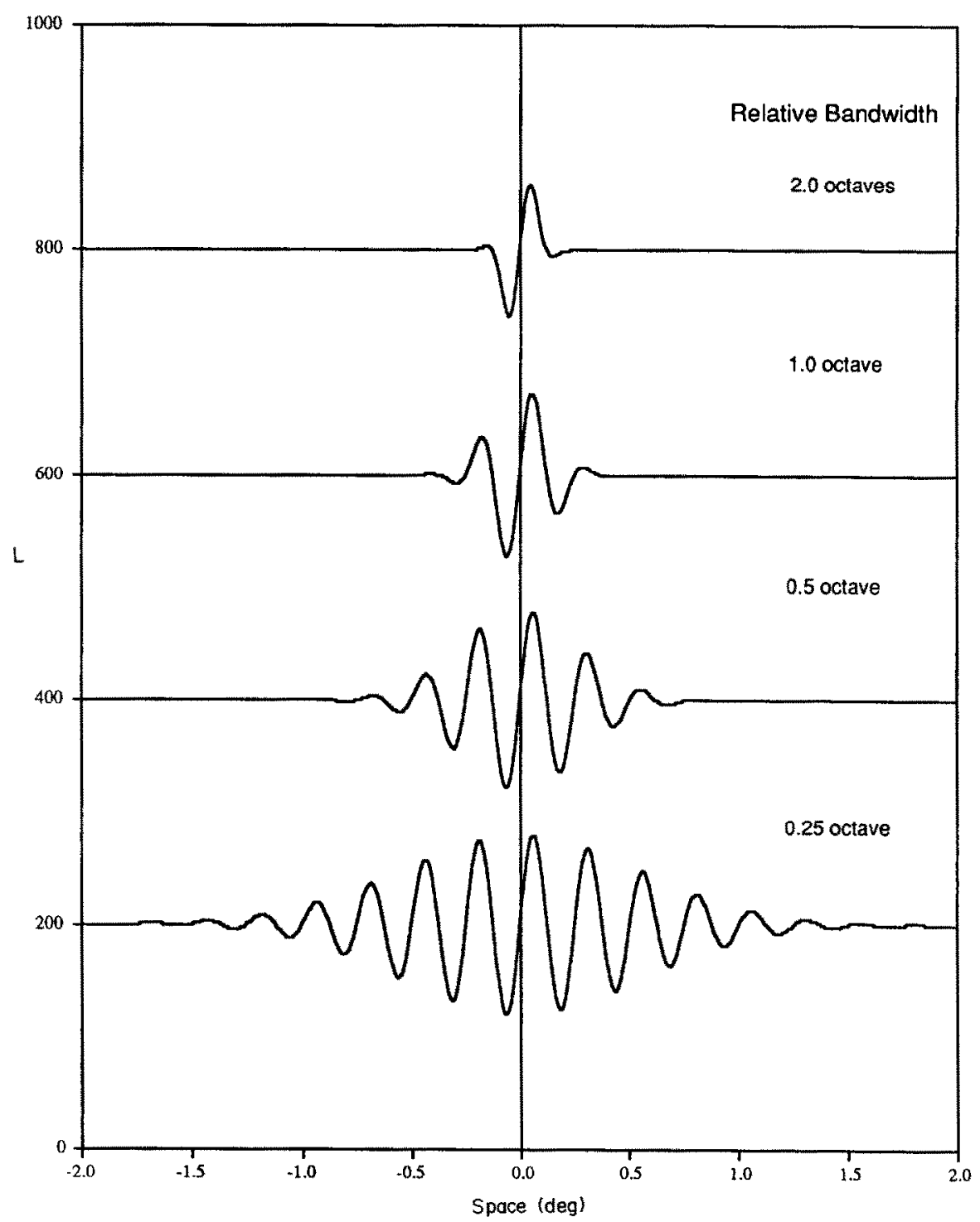

FIGURE 1. Luminance profiles of examples of the "Gaussian-windowed" stimulus patterns used. The luminance of the grating pattern, $L$, in $\mathrm{cd} / \mathrm{m}^{2}$, is shown as a function of space with respect to the center of the display $(0 \mathrm{deg})$. Gabor stimuli with a center spatial frequency of $4 \mathrm{c} / \mathrm{deg}$ and 4 different bandwidths $(0.25,0.5,1$ and 2 octaves) are shown. In the experiments, the spatial phase of the grating with respect to the center of the Gaussian envelope varied randomly from presentation to presentation. The actual mean luminance of the stimuli was $200 \mathrm{~cd} / \mathrm{m}^{2}$ and the modulation depth (contrast) varied from 0.01 to 0.16 .

\section{Procedure: Experiment 2}

Observers monocularly viewed the gratings at a distance of $1.14 \mathrm{~m}$ from the display. The display, subtending $15 \times 10 \mathrm{deg}$ at this distance, was surrounded by the same semi-circular, back-illuminated Plexiglass screen, which had a mean luminance of $100 \mathrm{~cd} / \mathrm{m}^{2}$. Viewing distance and head orientation was hold constant by having observers rest their chin on a chin-forehead rest. As in Experiment 1, the observers responded in a two-interval forced-choice paradigm which of two gratings had the higher spatial frequency. The retinal eccentricity of the Gaussian-windowed sinewave gratings was varied by having the subjects fixate a small black Letraset circle positioned either at the center or to the left of the center of the display, and the Gabor targets were presented either at the center or to the right of the center of the display. Both observers used their left eye. Their right eye was occluded with an eye patch. The location of the center of the Gaussian-truncated sinewave grating was either at the point of fixation (i.e. zero eccentricity) or $1,2,4,6,8,12$ or 20 deg eccentric of fixation along the horizontal meridian.

In separate experiments we determined the contrast thresholds for the same stimuli presented at the various retinal locations. Subjects pressed one of two buttons to signal in which of two intervals a low contrast grating was presented. The contrast of the grating was controlled on a 20-step staircase using the same Best-PEST algorithm. The upper and lower limits of the contrast range, differing by a factor of 10 , were set so that the expected threshold level was approximately midway between these bounds. A maximum-likelihood estimate of the detection threshold was determined after $\mathbf{4 0}$ trials.

\section{Observers}

Two male adults (the author MWG and a trained subject JO) served as observers. Both were corrected for their myopic and astigmatic refractive errors. The 


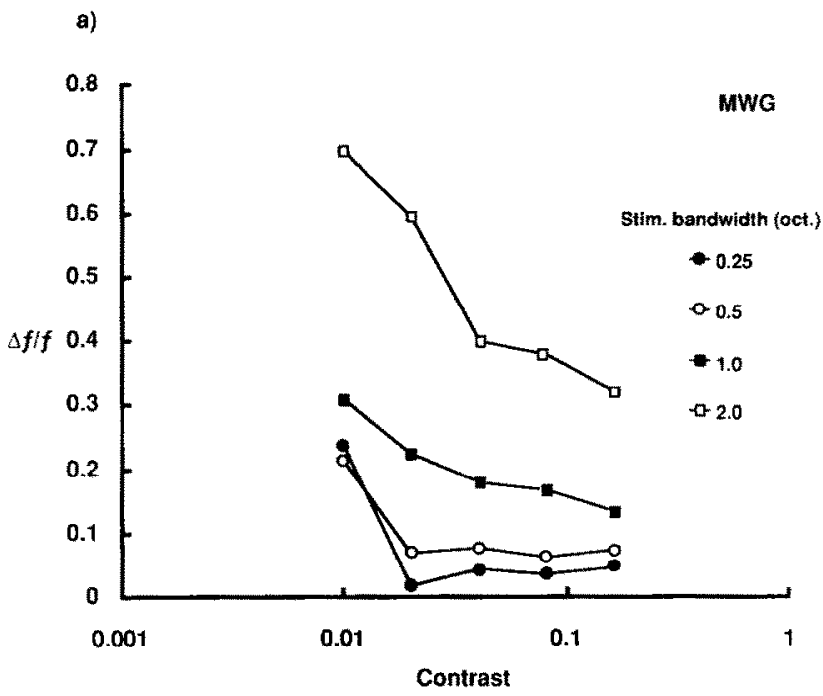

b)

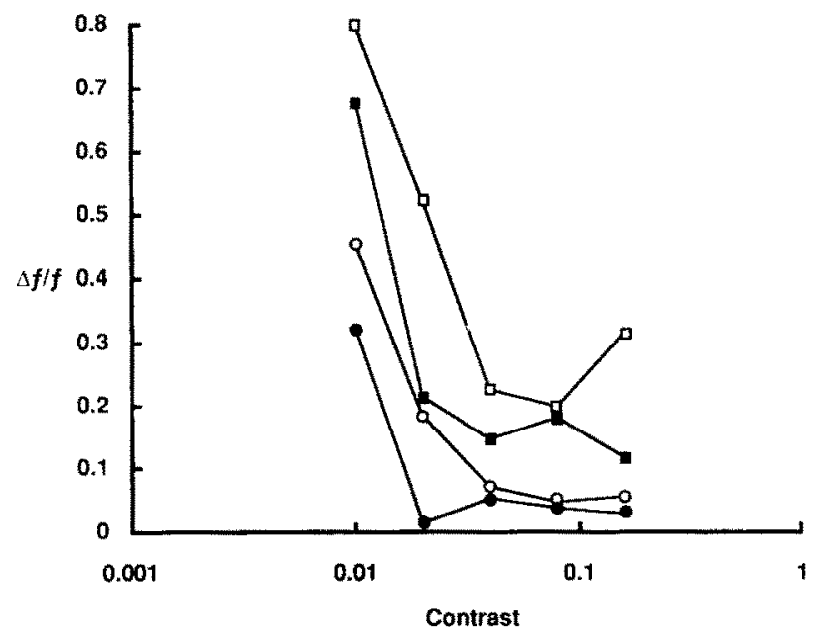

FIGURE 2. Spatial-frequency discrimination thresholds versus stimulus contrast. The Weber fraction $\Delta f / f$ is plotted as a function of the contrast of the Gabor targets. The parameter is the spatial-frequency bandwidth of the Gaussian-truncated grating (see inset). Symbols show the mean values of 3 runs. Results from observers MWG are shown in (a) and those for subject JO are shown in (b).

observers have had considerable experience in similar psychophysical tasks; JO was generally uninformed with respect to the experimental aims. Experiment 1 was conducted prior to Experiment 2. Both subjects were given a few training runs before each experiment to become acquainted with the procedure and task, the results of which are not included in the analysis.

\section{RESULTS}

Experiment 1: Spatial-frequency discrimination vs stimulus contrast and bandwidth

The results of the first experiment are shown in Fig. 2 for the two observers. The Weber fraction of spatialfrequency discrimination $(\Delta f / f)$ is plotted as a function of stimulus contrast. The reference spatial frequency in this experiment was $4 \mathrm{c} / \mathrm{deg}$. The different symbols present the results for the different bandwidth stimuli (see inset). The results for observer MWG are shown in
Fig. 2(a) and those for JO in Fig. 2(b). Spatial-frequency discrimination thresholds decrease with increasing stimulus contrast. As the stimulus bandwidth was increased the curves shifted upwards and the dependency on stimulus contrast also increased. Note that for the narrower stimulus bandwidths of 0.25 and 0.5 octave, spatial frequency discrimination is fairly independent of contrast, except for the lowest contrasts tested. MWG exhibits discrimination thresholds which are independent of contrast levels of 0.02 and above, whereas JO shows such independence for contrast levels of 0.04 and above. Thresholds are, on the other hand, markedly dependent on contrast for the broad-band stimuli. For the 2 octave stimulus, for example, thresholds decrease from 0.7 and 0.8 for 0.01 contrast levels to 0.3 for 0.16 contrast level for observer MWG and $\mathrm{JO}$, respectively. Results of a 2-way analysis of variance of the mean threshold values revealed a significant main effect
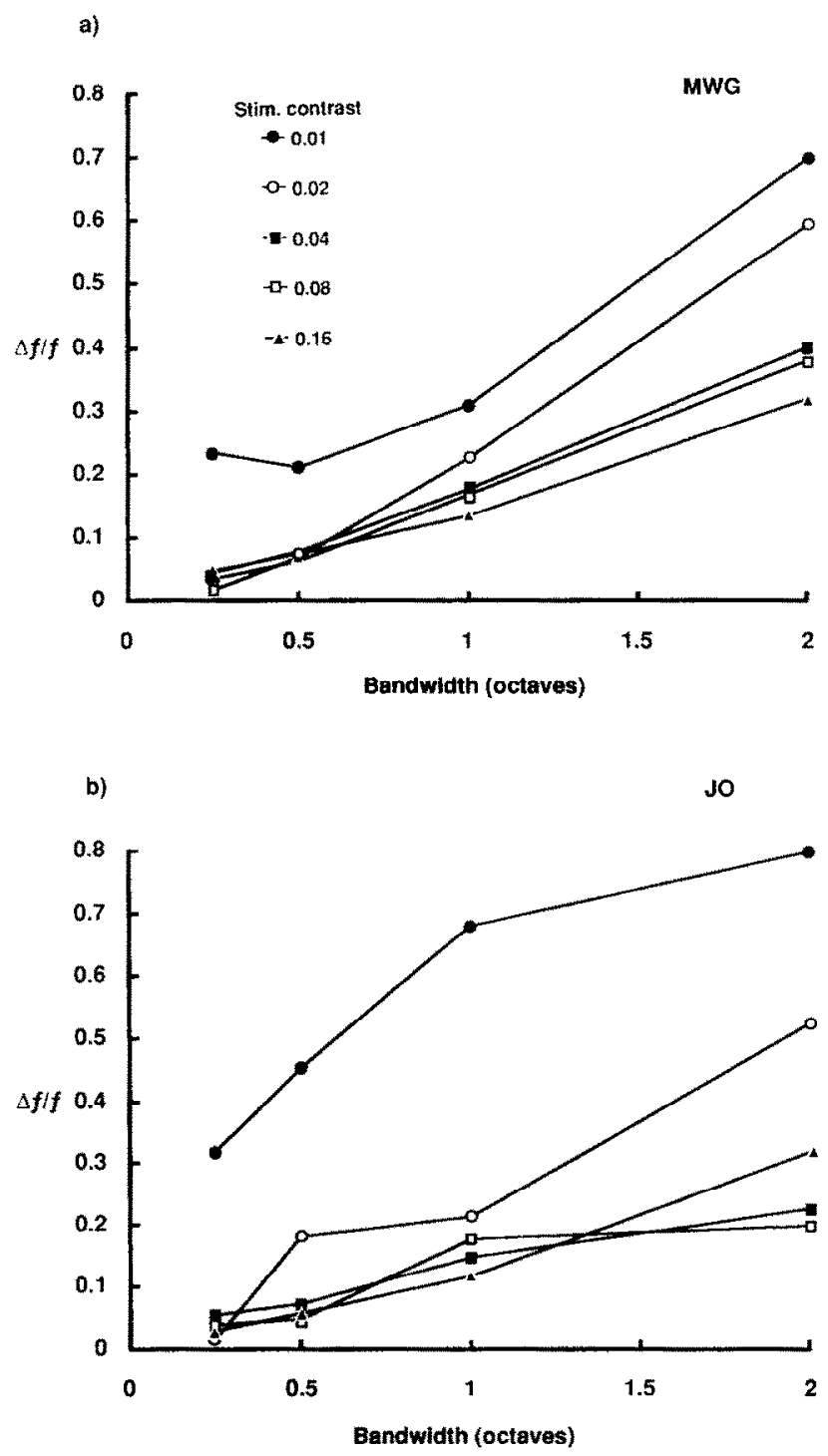

FIGURE 3. Spatial-frequency discrimination thresholds versus stimulus bandwidth. The Weber fraction $\Delta f / f$ is replotted as a function of the spatial-frequency bandwidth of the Gaussian-windowed sinewave gratings. Results are taken from Fig. 2. The parameter is the contrast of the gratings (see inset). Symbols show the mean values of 3 runs Results from observers MWG are shown in (a) and those for subject JO are shown in (b). 
a)

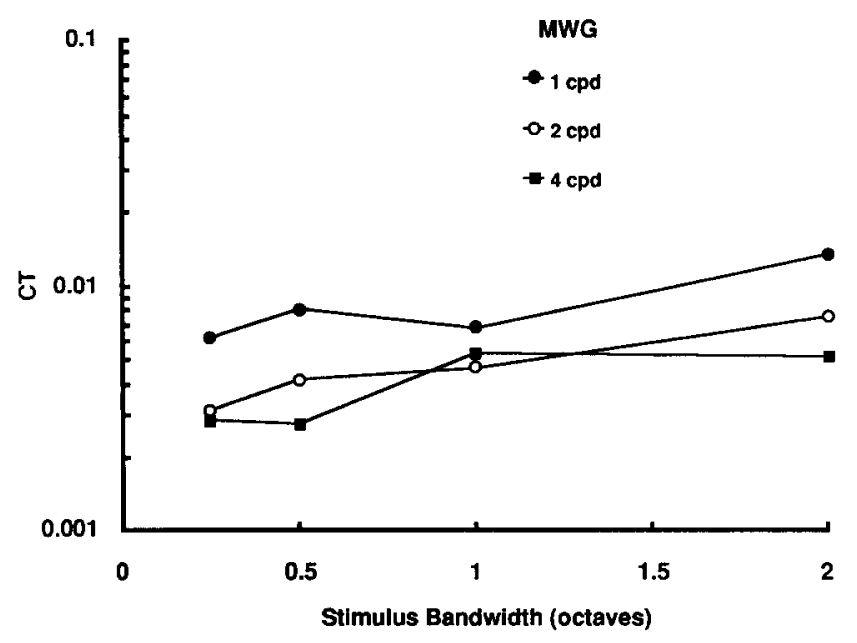

b)

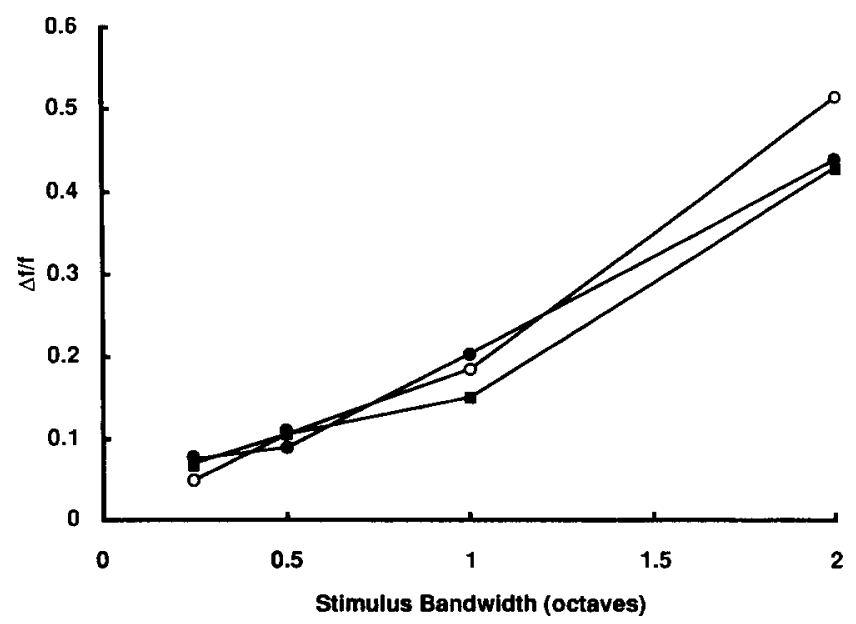

FIGURE 4. Contrast thresholds are shown in (a) for three reference spatial frequencies as a function of the stimulus spatial frequency bandwidth. (b) Spatial frequency discrimination thresholds are shown as a function of the relative spatial frequency bandwidth of the Gabor stimuli. The stimulus contrast was adjusted to remain 5 times higher than contrast threshold for the same stimuli used in the experiment shown in (a). The parameter is the reference spatial frequency of the gratings (see inset). Results are shown for subject MWG.

of contrast $\left(F_{4,20}=5.36, P<0.005\right)$ and bandwidth $\left(F_{3,20}=7.14, P<0.002\right)$ on discrimination thresholds. There was no significant interaction $\left(F_{12,20}=0.17\right.$, N.S. $)$ between these factors, indicating that the differences in the shape of these curves were not significant.

The results shown in Fig. 2 indicate that the bandwidth of the Gaussian-truncated sinewave gratings influenced the spatial-frequency discrimination threshold. In order to visualize this effect more clearly we replotted these results showing $\Delta f / f$ as a function of stimulus bandwidth (Fig. 3). The different symbols now represent the results for the various contrast levels (see inset). For both observers, $\Delta f / f$ increases with stimulus bandwidth. Furthermore, $\Delta f / f$ converges below a bandwidth of 0.5 octave, except for the lowest contrast value used (0.01). This convergence reflects the contrast constancy of discrimination thresholds for narrow-band stimuli. VR 32/2 - D
The results shown in Figs 2 and 3 clearly indicate that the effects of stimulus contrast and stimulus bandwidth jointly act to set the limits of spatial-frequency discrimination in the visual system. We next wanted to compare the effect of increasing stimulus bandwidth on spatialfrequency discrimination thresholds for different reference spatial frequencies. Since the contrast threshold varies with spatial frequency and with the spatialfrequency bandwidth (or space constant) of the gratings (Robson \& Graham, 1981; du Buf, 1987), we first measured contrast detection thresholds for the Gaussian-truncated gratings used in the experiments. The results are shown in Fig. 4(a), in which we plot contrast thresholds as a function of the stimulus bandwidth. The different symbols present the findings for the different spatial frequencies tested $(1,2$ and $4 \mathrm{c} / \mathrm{deg}$, see inset). Contrast thresholds increase slightly with increasing stimulus bandwidth. The effects of stimulus bandwidth and reference spatial frequency on detection threshold were tested for their statistical significance in a two-way analyses of variance. The effects of stimulus bandwidth $\left(F_{3,9}=1.17\right.$, N.S.) and reference spatial frequency $\left(F_{2,9}=1.46\right.$, N.S. $)$ on detection thresholds were not significant.

Figure 4(b) presents the results of the experiments in which we measured spatial-frequency discrimination thresholds for gratings having a constant suprathreshold contrast level. We chose to set the contrast in these experiments at a factor of 5 above the respective detection thresholds. It has already been shown that spatial-frequency discrimination thresholds become independent of contrast at this suprathreshold contrast level (Thomas, 1983; Burbeck, 1987), which is also indicated by the results shown in Fig. 2. Again, the different symbols present the results for the different reference spatial frequencies (see inset). The results in Fig. 4(b) reveal that $\Delta f / f$ increases almost proportionally to increasing stimulus bandwidth. The slope and magnitude of $\Delta f / f$, however, do not depend on the reference spatial frequency. The effects of stimulus bandwidth and reference spatial frequency on detection threshold were tested for their statistical significance in a two-way analyses of variance. The effect of stimulus bandwidth $\left(F_{3,12}=65.3, P<0.0001\right)$ but not reference spatial frequency $\left(F_{2.12}=0.42\right.$, N.S. $)$ was significant. The interaction between stimulus bandwidth and reference spatial frequency was not significant $\left(F_{6,12}=0.57\right.$, N.S. $)$, suggesting that the effect of relative stimulus bandwidth is independent of the reference spatial frequency.

Experiment 2: Contrast thresholds and spatial-frequency discrimination thresholds as a function of retinal eccentricity

The results of Experiment 2, in which we measured the effect of retinal eccentricity on contrast-detection and spatial-frequency discrimination thresholds for the Gaussian-truncated gratings, are shown in Figs 5 and 6. In Fig. 5, contrast threshold is plotted as a function of the retinal eccentricity at which the Gaussian-windowed gratings were centered. The parameter is the spatial 


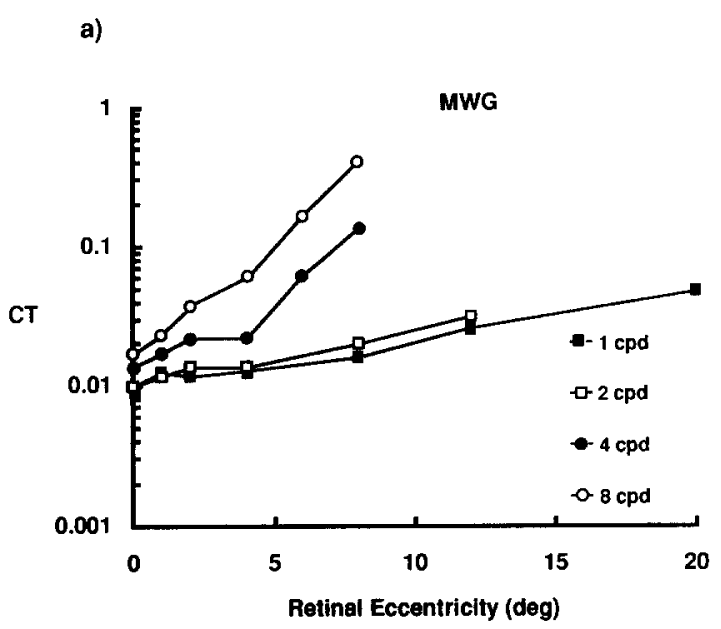

b)

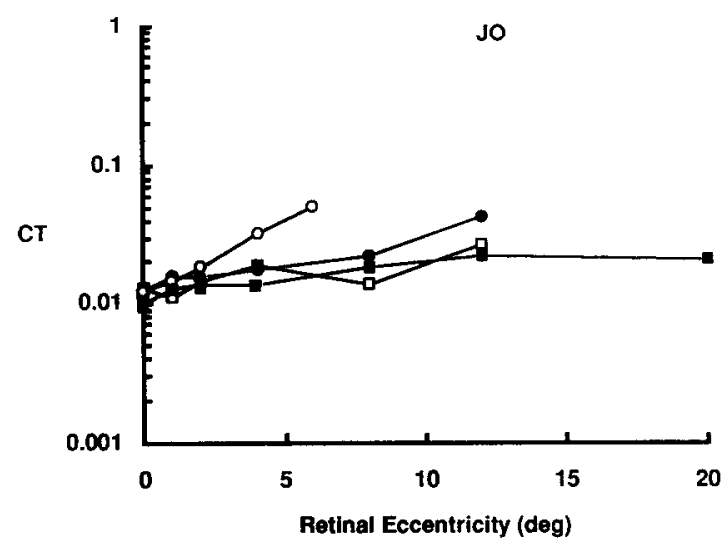

FIGURE 5. Contrast thresholds for Gaussian-truncated sinewave gratings are shown as a function of the retinal eccentricity at which they were presented along the horizontal meridian. Results for observer MWG are shown in (a), those for JO in (b).

frequency of the gratings, which varied from 1 to $8 \mathrm{c} / \mathrm{deg}$ (see inset). The spatial frequency bandwidth of the test gratings was held constant at 0.5 octave. Results for observer MWG are shown in Fig. 5(a) and those for observer JO are presented in Fig. 5(b). Contrast detection thresholds increased with increasing retinal eccentricity, in agreement with earlier studies (Hilz \& Cavonius, 1974; Koenderink, Bouman, Bueno der Mesquita \& Slappendel, 1978a,b; Virsu \& Rovamo, 1979). Note that thresholds are slightly higher in this experiment for central fixation, which is most likely a result of monocular viewing. A two-way analysis of variance revealed significant main effects of spatial frequency $\left(F_{3,20}=14.9, P<0.0001\right)$ and retinal eccentricity $\left(F_{4.20}=10.0, P<0.0001\right)$ on the logarithm of the mean contrast thresholds. The interaction term between these main effects $\left(F_{12,20}=1.4\right.$, N.S. $)$ was, however, not significant.

The results in Fig. 5 served as baseline data for our experiments concerned with the changes in spatialfrequency discrimination performance with increasing retinal eccentricity. Two cautions were taken in designing these experiments. (1) We kept the spatial-frequency bandwidth of the Gaussian-truncated sinewave stimuli constant by adjusting the size of the Gaussian envelope to correspond to the spatial frequency of the grating. (2) By adjusting the contrast of the grating stimuli to correspond to a value 5 times greater than contrast detection threshold, we can exclude the possibility that changes in discrimination threshold are related to the visibility of the gratings. Thus, changes in discrimination threshold with increasing retinal eccentricity and spatial frequency for these stimuli should reflect changes in the spatial-frequency selectivity and distribution of the underlying neural mechanisms.

The results of the experiments are shown in Fig. 6 . Spatial frequency discrimination threshold is plotted as a function of retinal eccentricity for Gaussian-windowed sinewave gratings having a 0.5 octave bandwidth. The different symbols show the results for the different reference frequencies (see inset). For all spatial frequencies tested, spatial frequency discrimination threshold
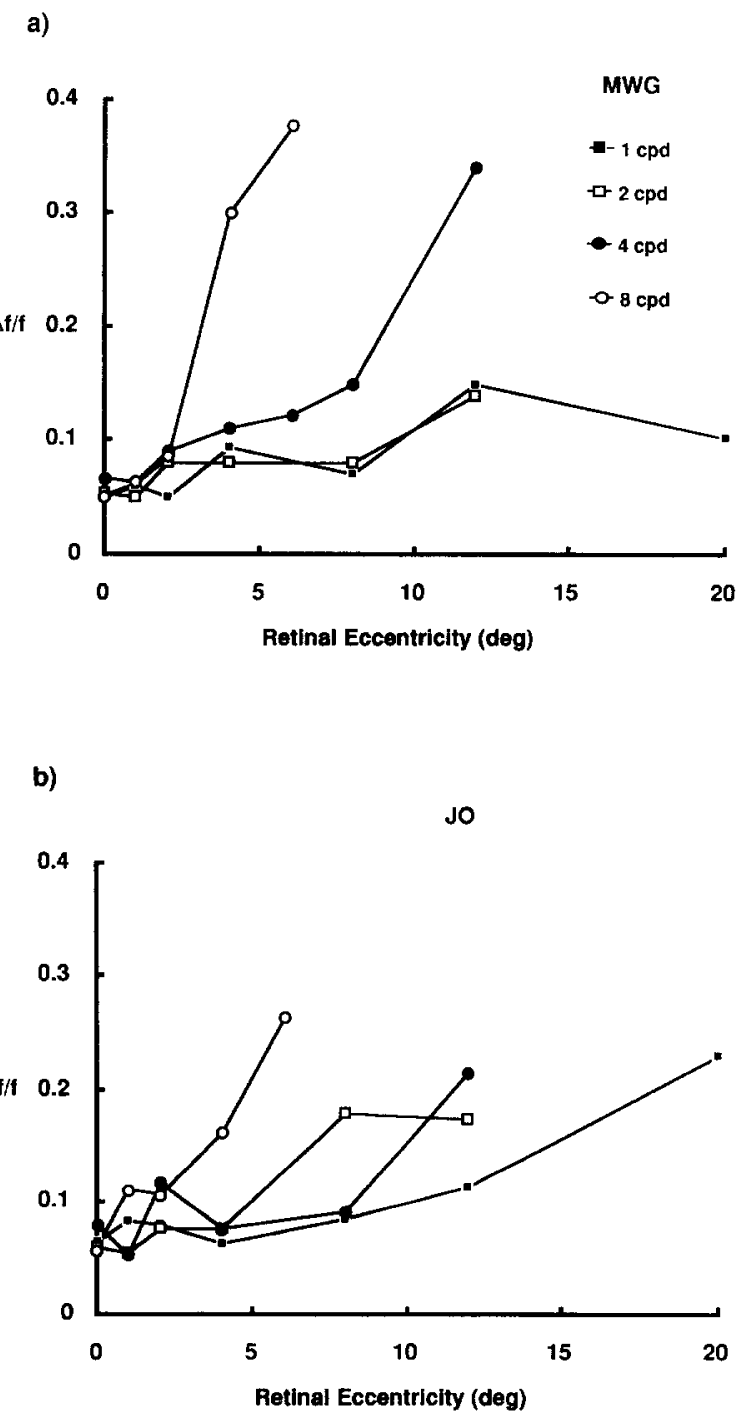

FIGURE 6. Spatial-frequency discrimination thresholds are shown as a function of the retinal eccentricity at which they were presented along the horizontal meridian. The stimuli were Gaussian-truncated sinewave gratings, with a spatial-frequency bandwidth of 0.5 octave and a contrast that was 5 times greater than detection threshold. Results for observer MWG are shown in (a), those for JO in (b). 


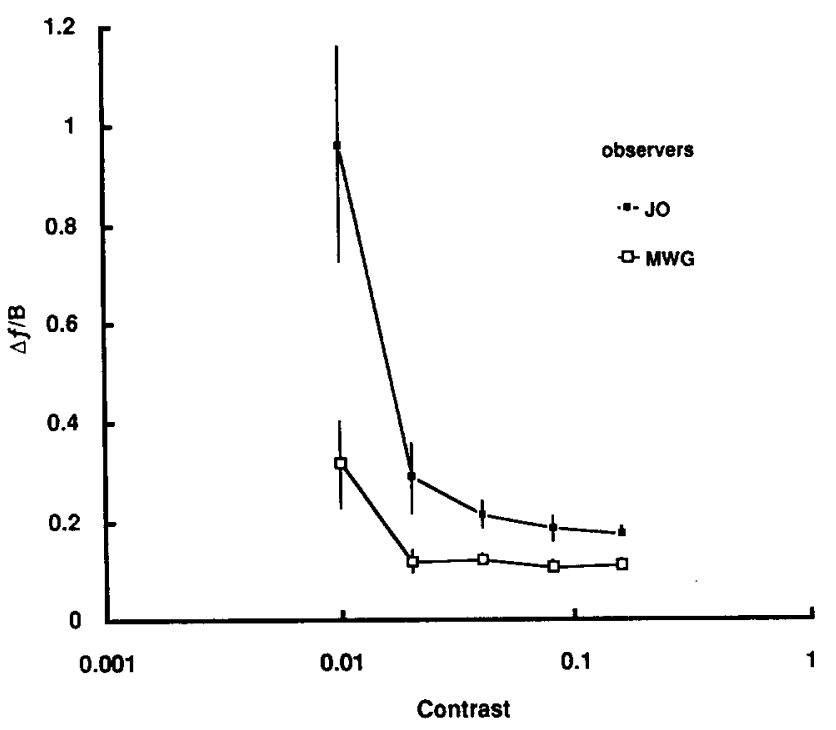

FIGURE 7. The results in Fig. 2 have been normalized by relating the spatial frequency difference (in octaves) $\Delta f$ to the relative spatial-frequency bandwidth $B$ (in octaves) of the Gaussian-windowed sinewave gratings. The ratio $\Delta f / B$ is plotted as a function of the contrast of the stimuli and error bars show $\pm 2 S E$. The results for observer MWG are shown by the open symbols and those for JO by solid symbols.

increases with increasing eccentricity. The rate of threshold change is, moreover, dependent on the spatial frequency of the reference gratings. For central presentation, $\Delta f / f$ is largely independent of the reference spatial frequency. The Weber fraction varies around 0.05 , which is in good agreement with values reported in the literature (Campbell et al., 1970; Mayer \& Kim, 1986). Discrimination thresholds for the different reference frequencies diverge as the Gabor targets are positioned more and more in the peripheral visual field. For the 1 and $2 \mathrm{c} /$ deg reference spatial frequencies, $\Delta f / f$ increases gradually from 0.05 to approx. 0.2 at $20 \mathrm{deg}$ eccentricity. The change in discrimination thresholds is much more pronounced for the medium-to-high spatial frequencies ( 4 and $8 \mathrm{c} / \mathrm{deg}$ ), which increased from 0.05 to approx. 0.35 (for observer MWG) and 0.27 (for observer JO) at $8 \mathrm{deg}$ retinal eccentricity. Note also that the individual differences apparent in the contrast detection vs eccentricity functions (Fig. 5) are reflected in the changes in discrimination threshold with increasing eccentricity. Observer MWG exhibits more pronounced changes in detection and discrimination thresholds with increasing retinal eccentricity. A two-way analysis of variance revealed significant main effects of reference spatial frequency $\left(F_{3.20}=12.8, P<0.001\right)$ and retinal eccentricity $\left(F_{4,20}=10.5, \quad P<0.0001\right)$ on $\Delta f / f$. The interaction between these main effects is also highly significant $\left(F_{12,20}=3.96, P<0.004\right)$, suggesting that the rate of change in discrimination threshold with increasing eccentricity varies for different reference spatial frequencies.

\section{DISCUSSION}

The results of Experiment 1 indicate that stimulus contrast and stimulus bandwidth interact to determine the discrimination threshold for spatial frequency. By increasing the spatial-frequency bandwidth of the Gaussian-windowed gratings, we decrease the precision with which the human visual system is capable of discriminating between gratings which differ in their spatial frequency i.e. the asymptotic value of $\Delta f / f$ vs log contrast increases. Viewed in the spatial-frequency domain, the effect of decreasing the space constant of the Gaussian envelope is equivalent to adding higher and lower spectral components to the frequency spectrum of the grating. Spatial frequency discrimination thresholds exhibit a substantial dependency on contrast when the bandwidth of the Gaussian-windowed gratings is 1 octave or greater. Howard $(1987,1989)$ has shown that the perceived spatial frequency of Gabor stimuli is altered when the spatial-frequency bandwidth of these stimuli is increased. Using a signal-detection approach, Howard and Thomas (personal communication) have independently found that the accuracy of spatial frequency discrimination is also reduced for stimuli with increased spatial-frequency bandwidths. Heeley and Thompson (1989) found that spatial frequency information can be integrated over 7-8 cycles of their high-contrast, grating patterns, and that this value is constant over a large range of reference spatial frequencies. Hirsch and Hylton (1982) reported a value of 2-3 cycles, over which spatial frequency information can be integrated. Differences in the stimuli used in these studies (spatial phase discontinuities vs abrupt truncation) and the present findings (Gaussian truncation) could be responsible for these differences.

Another way of expressing the ability of the visual system to discriminate the spatial frequency of bandlimited periodic patterns would be to relate the threshold spatial frequency difference to the stimulus bandwidth. With this in mind, we plotted $\Delta f / B$ as a function of contrast, where $\Delta f$ is the threshold spatial frequency difference (in octaves) and $B$ is the relative spatial frequency bandwidth (in octaves) of the Gaussianwindowed gratings. The results of this analysis are shown in Fig. 7 for subjects MWG and JO and are the mean $\pm 2 \mathrm{SE}$ of the discrimination threshold data from Fig. 2, averaged over the different bandwidth conditions. The value $\Delta f / B$ follows a single, monotonically decreasing function of stimulus contrast, which has an asymptotic value between 0.1 and 0.2 for our two observers. Note that, although these functions have a similar shape, the magnitude of $\Delta f / B$ for observer JO is higher than for observer MWG. Such individual differences could reflect differences in experience with discrimination judgments, MWG having more experience than JO.

The results of Experiment 2 indicate that gratings presented in the near periphery are discriminated with much less accuracy than those presented in the central visual field. The eccentricity-dependent change in the spatial-frequency discrimination threshold was found to depend on the reference spatial frequency of the gratings: $\Delta f / f$ changed more rapidly with retinal eccentricity for medium-high spatial frequency $(\geqslant 4 \mathrm{c} / \mathrm{deg})$ than for low spatial frequencies $(\leqslant 2 \mathrm{c} / \mathrm{deg})$. 
a)

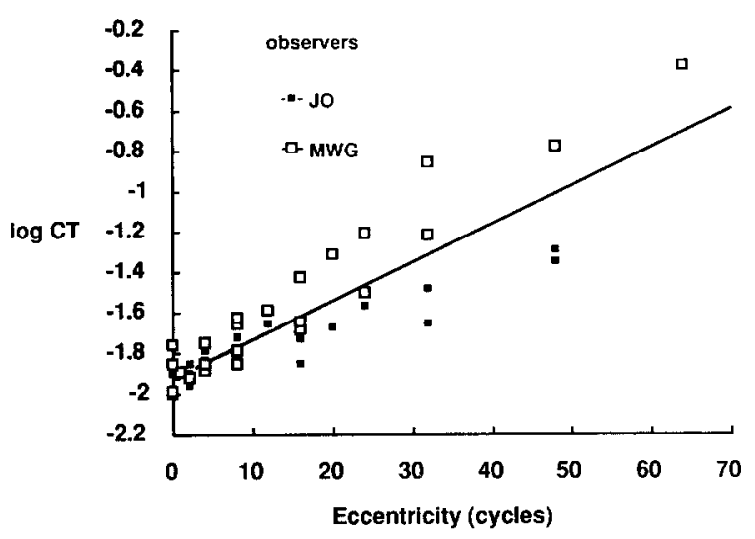

b)

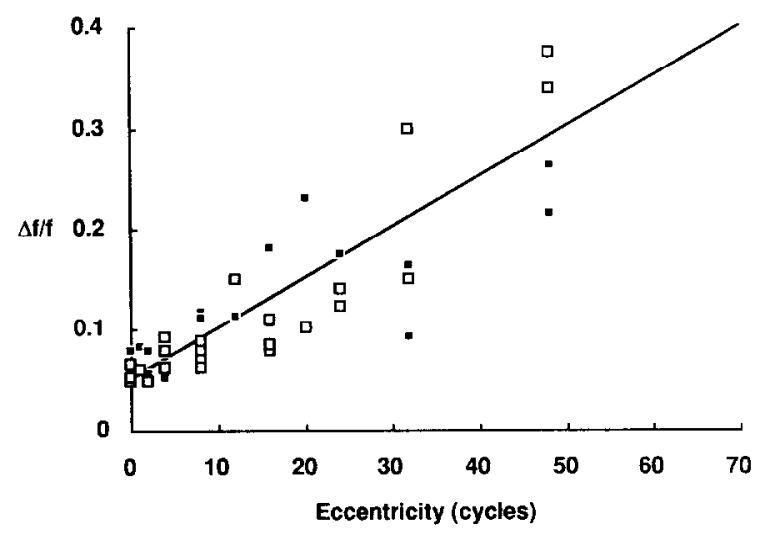

FIGURE 8. The results of Experiment 2 are replotted as a function of the number of cycles of the grating by which the stimulus is offset from the fixation point along the horizontal meridian. Panel a shows the results for log contrast threshold and panel $b$ presents the results for $\Delta f / f$. Solid symbols give the findings for subject JO and open symbols for subject MWG. The straight lines present the least-squares best fit of the regression of log threshold (panel a) or $\delta f / f$ (panel b) on eccentricity (defined in cycles of the grating patch).

As we adjusted the contrast of the gratings to remain a constant factor above the corresponding threshold detection level, the findings cannot be accounted for solely by considering the change in sensitivity to different spatial frequencics with increasing retinal cccentricity. They also cannot be accounted for by changes in the spatial-frequency bandwidth of the grating stimuli that often accompany changes in spatial frequency, since we used constant bandwidth stimuli ( 0.5 octave). As also demonstrated in the results of Experiment 1, discrimination performance in the central visual field is good $(\leqslant 0.05)$ for stimuli having a bandwidth of 0.5 octave and a suprathreshold contrast level. Performance for peripherally presented stimuli falls off with increasing eccentricity.

Thomas (1987) has reported that the ratio between the normalized probability that a grating is correctly identified and/or detected (I/D ratio) varies with retinal eccentricity. For low spatial frequencies ( 1 and $1.5 \mathrm{c} / \mathrm{deg}$ ) no significant effect of retinal eccentricity could be found in the mean $\mathrm{I} / \mathrm{D}$ ratios of three subjects, although individual differences were evident. For medium $(4-6 \mathrm{c} / \mathrm{deg})$ and high $(7-10 \mathrm{c} / \mathrm{deg})$ spatial frequencies the I/D ratios declined with retinal eccentricities exceeding $5 \mathrm{deg}$. In these experiments, the gratings used had a low contrast and the spatial frequency of the test and reference gratings differed by a set amount (varying from 18 to $50 \%$ depending on the conditions used). The composite data presented by Thomas (1987) in his Fig. 2 are thus in close agreement with our findings shown in Fig. 6. The individual differences reported by Thomas (1987) are also evident in our results and may be related to the difficulty of making spatial-frequency discriminations for peripherally viewed stimuli, especially those having high spatial frequencies. Indeed, the fact that the average $\mathrm{I} / \mathrm{D}$ ratio approaches zero for the $8 \mathrm{c} / \mathrm{deg}$ reference gratings in the Thomas (1987) study suggests that, although these gratings were visible differences as large as $42.8 \%$ could not be reliably discriminated.

Other forms of visual performance have been shown to decrease with increasing retinal eccentricity: grating acuity and resolution (Weymouth, 1958; Westheimer, 1982), vernier acuity (Westheimer, 1982; Levi, Klein \& Aitsebaomo, 1985), spatial phase discrimination (Rentschler \& Treutwein, 1985; Bennett \& Banks, 1987) and stereo acuity (Fendick \& Westheimer, 1983). Changes in visual performance with retinal eccentricity have been related to cone density (Rolls \& Cowey; Perry \& Cowey, 1985), retinal ganglion cell density (Rolls \& Cowey, 1970; Perry \& Cowey, 1985) and cortical magnitication (Cowey \& Rolls, 1974; Virsu \& Rovamo, 1979; for a recent review see Wilson, Levi, Maffei, Rovamo \& DeValois, 1990). Since the physiological findings of Daniel and Whitteridge (1961), which indicate that the cortical representation of the central visual field is many times larger than an equal-sized area in the peripheral visual field, it has been tempting for investigators using psychophysical methods to account for eccentricity-dependent changes in visual performance solely in terms of the cortical magnification factor.

Robson and Graham (1981) introduced the idea of normalizing the effect of retinal eccentricity by expressing eccentricity in terms of the number of cycles by which the grating patch is offset from the fixation point. After such normalization the slope of the log contrast threshold vs retinal eccentricity functions have approximately the same slope for different spatial frequencies (Robson \& Graham, 1981; their Fig. 4). In Fig. 8, the results of Experiment 2 are shown for log contrast threshold (panel a) and $\Delta f / f$ (panel b) for the two subjects tested. These values are taken from Figs 5 and 6 and are replotted as a function of the number of cycles the given vertical grating strip was displaced from the fixation point along the horizontal meridian. After this normalization the thresholds for both log contrast threshold and $\Delta f / f$ functions fall more or less along a single regression line. The linear regression of log contrast threshold on eccentricity (cycles) has a slope of 0.019 and an intercept of $-1.921\left(R^{2}=0.781\right)$. Similarly, the regression of $\Delta f / f$ on eccentricity (cycles) has a slope of 0.005 and an intercept of $0.053\left(R^{2}=0.738\right)$, 
averaged over observers and spatial frequencies. These results suggest that spatial frequency discrimination thresholds increase with increasing retinal eccentricity, although the stimuli used were presented at a set factor above detection threshold. If the change in spatial scale is taken into account by normalizing eccentricity in terms of number of cycles, the rate of this change is similar across a fairly wide range of spatial frequencies.

In summary, the present findings indicate that the spatial-frequency discrimination threshold depends on stimulus contrast and stimulus bandwidth. Only for a small set of stimulus contrast and bandwidth values (i.e $>0.02$ and $<0.5$ octave) is the spatial frequency discrimination threshold independent of stimulus contrast. The independence of $\Delta f / f$ from stimulus contrast, as described recently by Bowne (1990) is, thus, more the exception to the rule, rather than the rule itself. We have also shown that the spatial-frequency discrimination threshold increases with retinal eccentricity and that the rate of this increase depends on the reference spatial frequency. Westheimer (1982) has pointed out that changes in performance with increasing retinal eccentricity depend on the task being performed. Visual acuity or the minimal angle of resolution changes less rapidly than does, for example, hyperacuity thresholds. We have shown here that, if eccentric fixation is defined in terms of the number of cycles of the grating stimulus, log contrast threshold and $\Delta f / f$ increase in a similar manner over a wide range of spatial frequencies.

\section{REFERENCES}

Bowne, S. F. (1990). Contrast discrimination cannot explain spatial frequency, orientation or temporal frequency discrimination. Vision Research, 30, 449-461

du Buf, J. M. H. (1987). Spatial characteristics of brightness and apparent-contrast perception. Dissertation: University of Eindhoven, Holland.

Burbeck, C. A. (1987). Locus of spatial-frequency discrimination. Journal of the Optical Society of America A, 4, 1807-1813.

Campbell, F. W., Nachmias, J. \& Jukes, J. (1970). Spatial-frequency discrimination in human vision. Journal of the Optical Society of America, 60, 555-559.

Cowey, A. \& Rolls, E. T. (1974). Human cortical magnification factor and its relation to visual acuity. Experimental Brain Research, 2l, 447-454.

Daniel, P. M. \& Whitteridge, D. (1961). The representation of the visual field on the cerebral cortex in monkeys. Journal of Physiology, 159, 203-221.

Fendick, M. \& Westheimer, G. (1983). Effects of practice and the separation of test targets on foveal and peripheral stereoacuity. Vision Research, 23, 145-150.

Gouled Smith, B. \& Thomas, J. P. (1989). Why are some spatial discriminations independent of contrast? Journal of the Optical Society of America A, 6, 713-724.

Heeley, D. W. \& Thompson, R. J. (1989). The effect of stationary, random phase discontinuities, on spatial frequency discrimination. Vision Research, 29, 497-504.

Hilz, R. \& Cavonius, C. R. (1974). Functional organization of the peripheral retina: Sensitivity to periodic stimuli. Vision Research, 14, 1333-1337.
Hirsch, J. \& Hylton, R. (1982). Limits of spatial-frequency discrimination as evidence of neural interpolation. Journal of the Optical Society of America, 72, 1367-1374.

Howard, E. (1987). Perceived spatial frequency is altered for spatially narrow Gabor functions. Investigative Ophthalmology and Visual Science (Supplement), 28, 357.

Howard, E. L. (1989). Perception of sinusoidal gratings as a function of gaussian truncation. Doctoral dissertation, University of California, Los Angeles.

Koenderink, J. J., Bouman, M. A., Bueno de Mesquita, A. E. \& Slappendel, S. (1978a). Perimetry of contrast detection thresholds of moving spatial sine wave patterns. I. The far peripheral visual field (eccentricity $0^{\circ}-50^{\circ}$ ). Journal of the Optical Society of America, 68, 845849.

Koenderink, J. J., Bouman, M. A., Bueno de Mesquita, A. E. \& Slappendel, S. 1978b). Perimetry of contrast detection thresholds of moving spatial sine wave patterns. II. The near peripheral visual field (eccentricity $0^{\circ}-8^{\circ}$ ). Journal of the Optical Society of America, 68, 850-854.

Levi, D. M., Klein, S. A. \& Aitsebaomo, A. P. (1985). Vernier acuity, crowding and cortical magnification. Vision Research, 25, 963-977.

Lieberman H. R. \& Pentland A. P. (1982). Microcomputer-based estimation of psychophysical thresholds: The best PEST. Behavioral Research Methods and Instrumentation, 14, 21-25.

Marcelja, S. (1980). Mathematical description of the responses of simple cortical cells. Journal of the Optical Society of America, 70, $1297-1300$.

Mayer, M. J. \& Kim, C. B. Y (1986). Smooth frequency discrimination functions for foveal, high-contrast, mid spatial frequencies. Journal of the Optical Society of America A, 3, 1957-1969.

Perry, H. V. \& Cowey, A. (1985). The ganglion cell and cone distributions in the monkey's retina: Implications for central magnification factors. Vision Research, 12, 1795-1810.

Rentschler, I. \& Treutwein, B. (1985). Loss of spatial phase relationships in the extrafoveal vision. Nature, London, 313, 308-310.

Robson, J. G. \& Graham, N. (1981). Probability summation and regional variation in contrast sensitivity across the visual field. Vision Research, 21, 409-418.

Rolls, E. T. \& Cowey, A. (1970). Topography of the retina and striate cortex and its relationship to visual acuity in rhesus monkeys and squirrel monkeys. Experimental Brain Research, 10, 298-310.

Thomas, J. P. (1983). Underlying psychometric function for detecting gratings and identifying spatial frequency. Journal of the Optical Society of America, 73, 751-758.

Thomas, J. P. (1987). Effect of eccentricity on the relationship between detection and identification. Journal of the Optical Society of America, $A, 4,1599-1605$.

Virsu, V. \& Rovamo, J. (1979). Visual resolution, contrast sensitivity and the cortical magnification factor. Experimental Brain Research, 37, 475-494.

Watson, A. B. \& Robson, J. G. (1981). Discrimination at threshold: Labelled detectors in human vision. Vision Research, 21, 1115-1122.

Westheimer, G. (1982). The spatial grain of the perifoveal visual field. Vision Research, 22, 157-162.

Weymouth, F. W. (1958). Visual sensory units and the minimal angle of resolution. American Journal of Ophthalmology, 46, 102-113.

Wilson, H. R., Levi, D., Maffei, L., Rovamo, J. \& DeValois, R. (1990). The perception of form: Retina to striate cortex. In Spillmann, L. \& Werner, J. S. (Eds) Visual perception: The neurophysiological foundations (pp. 231-272). New York: Academic Press.

Acknowledgements - This research was supported by the Deutsche Forschungsgemeinschaft (SFB 325, B4). The author thanks J. Obergfell-Fuchs for his careful observations, Drs $\mathbf{H}$. du Buf and J. P. Thomas for helpful advice, and $\mathrm{Dr}$ L. Spillmann for providing laboratory facilities. 